\title{
Metallic Glass in Two Dimensional Disordered Bose Systems; A Renormalization Group Approach
}

\author{
M. Crisan • D. Bodea • I. Grosu • I. Ţifrea
}

Received: 15 July 2007 / Accepted: 15 August 2007 / Published online: 28 September 2007

(C) Springer Science+Business Media, LLC 2007

\begin{abstract}
We consider the quantum critical properties of a disordered two dimensional Bose gas based on the renormalization group approach. To account for disorder, we consider that the Bose gas interacts with an external random field. The system's behavior in the critical region of the quantum phase transition is obtained in the universality class $z=2$ and includes contributions beyond the Gaussian approximation by considering interactions between bosonic degrees of freedom. The evaluation of the system's conductivity close to the transition point proves the occurrence of a nonzero conductivity (metallic-like) state in the system in the $\omega=0$ and $T \rightarrow 0 \mathrm{~K}$ limit.
\end{abstract}

\section{Introduction}

Recently, Phillips and Dalidovich [1] introduced the idea of a Bose metal in connection with the unusual physical properties observed in two-dimensional (2D) systems in the proximity of the insulator-superconductor quantum phase transition. Experimental data for such systems suggested the presence of a metallic-like state in the $T \rightarrow 0 \mathrm{~K}$ limit characterized by a nonzero dc conductivity [2-4]. The presence

M. Crisan ( $\varangle) \cdot$ D. Bodea $\cdot$ I. Grosu

Department of Physics, "Babeş-Bolyai”" University, 40084

Cluj-Napoca, Romania

e-mail: mcrisan@phys.ubbcluj.ro

M. Crisan · D. Bodea

Max Plank Institute for the Physics of Complex Systems, 01187

Dresden, Germany

I. Ţifrea

Department of Physics, California State University, Fullerton, CA 92834, USA of the metallic state is inconsistent with the electronic description used to analyze the occurrence of the insulating phase in 2D systems and apparently its origin is related to bosonic degrees of freedom $[1,5]$. One possible way to describe this phenomena was proposed by Das and Doniach [5] who considered the metallic phase to be a uniform phase lacking both phase and charge order. This phase presents translational and rotational invariance and has to appear at finite temperature, as at $T=0 \mathrm{~K}$ there is no phase transition which is lacking both charge and phase order [6]. The occurrence of a metallic phase in a disorder 2D bosonic system has been predicted in the insulator-superconductor transition by Wagenblastl et al. [7], but only at the separatrix line.

In this paper we present a model for the 2D disordered Bose system which can be treated with the Renormalization Group (RG) method. The results we obtained are similar to the one of Dalidovich and Phillips [8] but have the advantage that contributions beyond the Gaussian approximation can be analyzed. The model was previously used to describe the spin glass state, disorder being introduced by a random field which can be connected to the effective order parameter of the spin glass state $[10,11]$. The quantum version of the model was analyzed by Busiello et al. $[12,13]$ to study the critical behavior of the interacting disordered Bose system. Our analysis focuses on a 2D disordered Bose system with a dynamical critical exponent $z=2$, situation in which we can solve exactly the RG equations. This allows us to study the influence of the Edwards-Anderson parameter associated with the spin glass state $q$ and the fluctuations interaction $u_{0}$ near the fixed point $T=0$ on the system's physical properties. Accordingly, our results will be relevant beyond the Gaussian approximation used previously. Our main focus is on the estimation of the dc conductivity for a disordered 2D Bose system. Transport theory in the critical region is not trivial as the limits $\sigma(T=0, \omega \rightarrow 0)$ and 
$\sigma(\omega=0, T \rightarrow 0)$ are not equal [14-16]. The relevant quantity for the dc conductivity in the vicinity of the critical point is $\sigma(\omega=0, T \rightarrow 0)$. We will calculate the conductivity in the critical region following the same method we apply recently for the study of the conductivity in $d$-wave superconductors near the critical disorder [17]. Our results for the dc conductivity will contain corrections to the Gaussian behavior which will depend both on the Edwards-Anderson spin glass parameter $q$ and bosonic fluctuations interaction $u_{0}$.

\section{Renormalization Group Method}

To analyze the possibility of a metallic state in a Bose gas Dalidovich and Phillips [8] considered a model based on the quantum version of the spin glass state which can be described using the replica trick method. Their main result is that the glass phase which may interrupt the superfluidinsulator phase transition is a metallic phase with a welldefined dc conductivity in the $T \rightarrow 0$ limit. The model has as a starting point an array of supercoducting islands described by a random Josephsson Hamiltonian, which was mapped in a disordered $X Y$ spin model. The Landau action for this problem can be obtained using the replica trick method to perform the average on the disordered state. The quadratic and quartic terms describe in the spin glass theory the interaction between spins that appears by the average procedure on the disordered state, and can be decoupled by introducing the auxiliary fields $Q_{\mu \nu}^{a b}\left(\mathbf{k}, \mathbf{k}^{\prime}, \tau, \tau^{\prime}\right)=\left\langle S_{\mu}^{a}(\mathbf{k}, \tau) \mathbf{S}_{\nu}^{\mathbf{b}}\left(\mathbf{k}^{\prime}, \tau^{\prime}\right)\right\rangle$ and $\Phi_{\mu}^{a}(\mathbf{k}, \tau)=\left\langle\mathbf{S}_{\mu}^{\mathbf{a}}(\mathbf{k}, \tau)\right\rangle$, where the superscriptions $a$ and $b$ represent the replica indices. A finite value of $\Phi_{\mu}^{a}$ is equivalent with the charge phase ordering in the condensate, and $\left\langle\Phi_{\mu}^{a}\right\rangle=0$ describe the disordered phase. For the quantum spin glass the diagonal elements of $Q\left(\mathbf{k}, \mathbf{k}^{\prime}, \tau, \tau^{\prime}\right)$, in the limit $\left|\tau-\tau^{\prime}\right| \rightarrow \infty$ are the effective Edwards-Anderson spin-glass order parameter [8,9]. The quantum decay of $Q_{\mu \mu}^{a a}(\tau)$ is like $\tau^{-2}$ and this gives rise to excitations which scales as $|\omega|$. The behavior of this parameter leads to the change of the critical dynamic exponent from $z=1$ to $z=2$ and the onset of overdamped dynamics. The free energy given in Ref. [8] contain a Gaussian and a quartic term as in the standard $\Phi^{4}$-theory, and the contribution which couples the charge and the glass degrees of freedom. The model was treated by the authors in the Gaussian approximation and the conductivity $\sigma(\omega=0, T \rightarrow 0)$ has been obtained to be finite, proving the possibility of the metallic state. However, it may be important to include terms beyond the Gaussian approximation as they can account for changes in the physical properties of the system.

In this section we will introduce our model for the 2D disordered Bose system and consider the quantum critical properties of the system by solving the RG equations. The model is equivalent to the one proposed by Dalidovich and
Phillips for the description of the metallic glass phase in the insulator-superconductor transition [8]. In our case, the value of the dynamical critical exponent is $z=2$ as it results from the renormalization procedure. Also, our analysis is carried beyond the Gaussian approximation as we considered for the characterization of the critical region the appropriate interactions between bosonic degrees of freedom.

Following Busiello et al. $[12,13]$ a $d$-dimensional Bose gas in the presence of disorder can be described by a Hamiltonian which includes coupling of the bosonic degrees of freedom to a random field $h(\mathbf{x})$ :

$$
\begin{aligned}
H= & \int d^{d} x \Phi^{\dagger}(\mathbf{x})\left[-\nabla^{2}+r_{o}\right] \Phi(\mathbf{x})+\frac{u_{0}}{4} \int d^{d} x|\Phi(\mathbf{x})|^{4} \\
& +\int d^{d} x\left[h(\mathbf{x}) \Phi^{\dagger}(\mathbf{x})+h^{\star}(\mathbf{x}) \Phi(\mathbf{x})\right]
\end{aligned}
$$

where $\Phi(\mathbf{x})$ represents the bosonic fields, $r_{0}$ the control parameter (for the standard case it is the chemical potential), and $u_{0}>0$ is the bare coupling constant describing the interaction between the bosonic degrees of freedom $\left(\hbar^{2}=2 m=1\right)$. The random field $h(\mathbf{x})$ is a Gaussian random variable with a Fourier transform $h(\mathbf{k})$ which satisfies:

$\langle h(\mathbf{k})\rangle=\left\langle h^{\star}(\mathbf{k})\right\rangle=0$,

$\left\langle h^{\star}(\mathbf{k}) h\left(\mathbf{k}^{\prime}\right)\right\rangle=\delta_{\mathbf{k}, \mathbf{k}^{\prime}} q$,

where $\langle\cdots\rangle$ denotes an average over the possible configurations of the random field and serves to introduce an effective Edwards-Anderson spin glass parameter, $q$ [9]. The system's action can be written as

$S[\Phi]=S^{(0)}[\Phi]+S^{(\mathrm{int})}[\Phi]$,

with the Gaussian contribution given by:

$$
\begin{aligned}
S^{(0)}[\Phi]= & \sum_{a, b=1}^{m} \sum_{k}\left[\left(r_{0}+k^{2}-i \omega_{n}\right) \delta_{a b}-\frac{q}{T} \delta_{\omega_{n}, 0}\right] \\
& \times \Phi_{a}(k) \Phi_{b}(k),
\end{aligned}
$$

and the interaction contribution by:

$$
\begin{aligned}
S^{(\mathrm{int})}[\Phi]= & \frac{u_{0}}{4} \sum_{a=1}^{m} \sum_{k_{1}} \cdots \sum_{k_{4}} \Phi_{a}\left(k_{1}\right) \cdots \Phi_{a}\left(k_{4}\right) \\
& \times \delta\left(k_{1}+\cdots+k_{4}\right) .
\end{aligned}
$$

Above $k \equiv\left(\mathbf{k}, \omega_{n}\right), \omega_{n}=2 \pi n T$ are the bosonic Matsubara frequencies, $\sum_{k} \rightarrow \sum_{\omega_{n}} \int d^{d} k /(2 \pi)^{d}$, and $m$ is the replica index from the standard spin-glass theory [9]. The Gaussian propagator of the system can be calculated in the limit $m \rightarrow 0$ as [1, 12]:

$$
G_{a, b}(k)=G_{0}(k) \delta_{a, b}+\beta G_{0}^{2}(k) q \delta_{\omega_{n}, 0},
$$


where $\beta=1 / T$ and $G_{0}\left(\mathbf{k}, \omega_{n}\right)=\left(r_{0}+k^{2}-i \omega_{n}\right)^{-1}$. Similar to Ref. [8], we also considered the replica symmetric case, but we do not have a term which couples the bosonic field $\Phi$ and the order parameter $q$. The model we consider is a simpler approximation as we use the free propagator with no Ohmic dissipative term, meaning that any time dependence of the effective parameter $q$ can be neglected. In this case, the effective parameter $q$ is considered to be only position dependent. The advantage is that we can treat the phase transition using the RG theory beyond the Gaussian approximation and prove the relevance of the effective parameter $q$ and of the interaction between the bosonic fluctuations on the system's properties.

The temperature-dependent RG flow equations were thereafter obtained following the standard procedure, $\Phi=$ $e^{l(d+z+2)} \Phi^{\prime}, k=k^{\prime} e^{-l}$, and $\omega=\omega^{\prime} e^{-l z}$, leading to

$\frac{d T(l)}{d l}=z T(l)$,

$\frac{d q(l)}{d l}=(2+z) q(l)$,

$\frac{d r(l)}{d l}=2 r(l)+u(l) F_{1}(l, T)+u(l) q(l) F_{2}(l)$,

and

$$
\begin{aligned}
\frac{d u(l)}{d l}= & {[4-(d+z)] u(l)-\frac{u^{2}(l)}{4}\left[8 F_{3}(l, T)+2 F_{4}(l, T)\right] } \\
& +5 u^{2}(l) q F_{5}(l) .
\end{aligned}
$$

In the above equations, $z=2$ and $d=2$. The functions $F_{i}(l)(i=2, \ldots, 5)$ can be calculated [12], however, only the function $F_{1}\left(T(l)=K_{2}[\exp (1 / T(l))-1]^{-1}\right.$ with $K_{2}=$ $1 /(2 \pi)$ is important because contains the relevant temperature parameter $T(l)$. The first two flow equations, i.e., (7-8), can be solved easily with $T(l)=T e^{2 l}$ and $q(l)=q e^{4 l}$ as solutions. To solve the other two flow equations, i.e., (9-10), we consider the low temperature limit and approximate $8 F_{3}+2 F_{4} \simeq K_{2}$, and $F_{5} \simeq K_{2}$. Using these results one finds

$u(l)=\frac{4}{K_{2}} \frac{1}{l+l_{0}+5 q\left(e^{4 l}-1\right)}$,

where $l_{0}=4 /\left(K_{2} u_{0}\right)$. The last flow equation to solve is the one for the control parameter, i.e., (9). Its general solution can be obtained if we consider $F_{2} \simeq K_{2}$ and search for a solution $r(l)=r_{0} \exp (2 l) h(l)$ as:

$r(l)=e^{2 l}\left[r_{0}+I_{1}(T)+4 q \int_{0}^{l} d l^{\prime} \frac{e^{2 l^{\prime}}}{l^{\prime}+l_{0}+5 q\left(e^{4 l}-1\right)}\right]$, where

$I_{1}(T)=4 \int_{0}^{l} d l^{\prime} \frac{u\left(l^{\prime}\right) \exp \left(-2 l^{\prime}\right)}{\exp \left[1 / T\left(l^{\prime}\right)-1\right]}$.

If one considers the low temperature limit $I_{1}(T) \simeq 0$, and for small values of the Edwards-Anderson spin-glass parameter $q$, (12) can be approximated as

$r(l)=e^{2 l}\left[r_{0}+4 q \int_{0}^{l} d l^{\prime} \frac{e^{2 l^{\prime}}}{l^{\prime}+l_{0}}\right]$.

The integral on the left-hand side of (14) can be calculated exactly in terms of the exponential-integral special function $E i(x)$ which thereafter can be approximated by its value in the large $x$ limit. Finally, the general solution for the last flow equation can be given as

$r(l) \simeq e^{2 l}\left[r_{0}+2 q\left(\frac{e^{2 l}}{l+l_{0}}-\frac{1}{l_{0}}\right)\right]$.

The renormalization procedure will be stopped at $l^{*}$ given by the condition $r\left(l^{\star}\right)=1$. This procedure gives the possibility to study the temperature influence on the system's properties in the critical region of the $z=2$ insulatorsuperconductor phase transition. In our case, the Gaussian fixed point $T=0$ is stable and we will expect to be able to evaluate also the role played by the interaction between fluctuations on the system's behavior. Technically, the equation we have to solve is very complicated, so an approximation is required for an estimation of $l^{*}$. We choose to solve the equation using consecutive iterations with the final solution

$e^{4 l^{\star}} \simeq\left(1+u_{0} q\right)^{2}$.

The system's physical properties in the critical region can be estimated if we consider all the solutions of the RG equations in the $l \rightarrow l^{*}$ limit. For example, the EdwardsAnderson spin-glass parameter will be $q(l)^{*}=q\left(1+u_{0} q\right)$, a value which considers the role played by interaction between the bosonic variable in the critical region.

\section{DC Conductivity}

In this section, we will evaluate the dc conductivity of the system in the low temperature limit. We will start from the Kubo formula, and thereafter consider the limit $\omega=0$ and $T \rightarrow 0$. To evaluate the system's transport properties in the critical region one has to use the scaling of the conductivity, which in the case of a two dimensional system take the simple form $\sigma(r, T, u, q)=\sigma\left(r\left(l^{*}\right), T\left(l^{*}\right), u\left(l^{*}\right), q\left(l^{*}\right)\right)$ [18]. The formalism we use is not different from the one in Ref. [8] as it relies on the same generalized Kubo formula used to calculate the transport properties in the replica-trick 
method. The advantage of our analysis is that we can consider the influence of the bosonic fluctuations interaction. The Edwards-Anderson spin-glass parameter is strongly dependent on the renormalization parameter, $l$, and in the critical region we can fix its value at $q\left(l^{*}\right)$, a procedure which will allow us to study the competition between disorder and fluctuations interaction in the occurrence of the metallic phase. On the other hand, the existence of the dissipation term in the noninteracting action is essential for the occurrence of the metallic state as it was first pointed out in Ref. [7]. However, if our model appear to be more treatable analytically by the RG method, the main approximation of the model remain the dissipative form of the free propagator $G_{0}\left(\mathbf{k}, \omega_{n}\right)$. Such a form of the free propagator can be easily obtained if we adopt a model similar with the one in Ref. [17], where the free propagator contains the scattering effects on the nonmagnetic impurities, or the coupling to an Ohmic heat bath [18]. This approximation is well justified by the results, and a RG-treatment containing a more complicated form of the action is difficult to be controlled. The higher order corrections mentioned in Ref. [8] lead to terms proportional to $u_{0} q^{2}$ which appear also in our calculations, but we cannot consider these corrections as having the same origin. In fact, the vertex corrections presented later by the authors in Ref. [8] leads to the same qualitative behavior for the $T=0$ case, the difference being the power of $r_{0}$ in the denominator, a result similar with the one presented in Ref. [17] for the case of a layered quasi-two dimensional superconductor.

To calculate the transport properties of the system we start from the general Kubo formula for a disordered system [8]:

$$
\begin{aligned}
\sigma\left(i \omega_{n}\right)= & \frac{8 e^{2}}{m \omega_{n}} T \sum_{a, b, \omega_{l}} \int \frac{d^{2} \mathbf{k}}{(2 \pi)^{2}}\left[G_{a, b}^{0}\left(\mathbf{k}, \omega_{l}\right) \delta_{a, b}\right. \\
& \left.-2 k_{x}^{2} G_{a, b}^{0}\left(\mathbf{k}, \omega_{l}\right) G_{a, b}^{0}\left(\mathbf{k}, \omega_{l}+\omega_{n}\right)\right],
\end{aligned}
$$

equation which can be simplified to the following form

$$
\begin{aligned}
\sigma\left(\omega_{n}\right)= & \frac{8 q\left(l^{\star}\right) e^{2}}{\omega_{n}} \int \frac{d^{2} \mathbf{k}}{(2 \pi)^{2}} k_{x}^{2} G_{0}^{2}(\mathbf{k}, 0) \\
& \times\left[G_{0}(\mathbf{k}, 0)-G_{0}\left(\mathbf{k}, \omega_{n}\right)\right] .
\end{aligned}
$$

The dc conductivity can be evaluated relatively easy using the dissipative form of the free propagator $G_{0}\left(\mathbf{k}, \omega_{n}\right)$ along with the renormalized value for the Edwards-Anderson spin-glass parameter, $q\left(l^{*}\right)$, as:

$\sigma(\omega=0, T \rightarrow 0)=\frac{4 e^{2}}{3} \frac{q\left(1+u_{0} q\right)^{2}}{r_{0}^{4}}$.

In the Gaussian approximation, i.e., for negligible fluctuations interaction $\left(u_{0} \rightarrow 0\right)$, our result reconfirm the result obtained by Dalidovich and Phillips [8]. A nonzero conductivity in the $\omega=0, T \rightarrow 0$ limit proves the existence of a metallic-like state in the insulator-superconductor phase transition. Such a metallic state is robust respect to the bosonic interactions in the system.

\section{Discussions}

In conclusion, we presented a calculation of the transport properties for a disordered bosonic system, namely, we evaluated the dc conductivity in the $T \rightarrow 0$ limit. Our simple model, which accounts for the static disorder in an interacting Bose gas, proved the existence of a metalliclike state $(\sigma(\omega=0, T \rightarrow 0) \neq 0)$ close to the $T=0$ insulator-superconducting phase transition. This state is in fact a bosonic metallic glass, predicted first by Dalidovich and Phillips [8]. The model we proposed considers effects beyond the Gaussian approximation, accounting for the interactions between bosonic fluctuations. The final conductivity expression given by (19) shows that the dissipation itself cannot induce a new state in the system, but disorder can drive the two dimensional interacting Bose system in a new state. The role played by the bosonic fluctuations interaction is reflected in a correction term to the system's dc conductivity of the order of $\left(1+u_{0} q\right)^{2}$, where $u_{0}$ is the bare interaction between bosons and $q$ the spin-glass EdwardsAnderson parameter. The analysis of the model was also performed by Busiello et al. [12, 13] using a $6-\epsilon$ expansion to discuss the system's properties in the critical region, with the conclusion that the resulting state is a simple spin glass. No analysis of the transport properties was performed in Refs. $[12,13]$.

We expect that the intermediate metallic state predicted for disordered 2D Bose gases to appear in $d$-wave superconductors containing nonmagnetic impurities. In such systems, disordered is responsible for an insulator-superconducting phase transition. The theoretical analysis of the dc conductivity near the critical concentration which destroys the $d$-waves superconducting state contains also the influence of the temperature on the metallic conductivity [17]. However, from the experimental point of view, the existence of a metallic glass phase in the critical region of the insulator-superconducting phase transition still needs to be confirmed [19].

Acknowledgements This work was partially supported by The Romanian National University Research Council. One of the authors (M.C.) is glad to thank Prof. Philip Phillips for stimulating discussions. M.C. is glad to thank Prof. Peter Fulde for his hospitality at The Max Planck Institute for the Physics of Complex Systems where the manuscript was finalized. 


\section{References}

1. Phillips, P., Dalidovich, D.: Science 302, 243 (2003)

2. Jaeger, H.M., Haviland, D.B., Orr, B.G., Goldman, A.M.: Phys. Rev. B 40, 182 (1989)

3. Ephron, D., Yazdani, A., Kapitulnik, A., Beasley, M.R.: Phys. Rev. Lett. 76, 1529 (1996)

4. Mason, N., Kapitulnik, A.: Phys. Rev. Lett. 82, 5341 (1999)

5. Das, D., Doniach, S.: Phys. Rev. B 60, 1261 (1999)

6. Fazio, R., Schön, G.: Phys. Rev. B 43, 5307 (1991)

7. Wagenblast, K.-H., van Otterlo, A., Schön, G., Zimanyi, G.T.: Phys. Rev. Lett. 78, 1779 (1997)

8. Dalidovich, D., Phillips, P.: Phys. Rev. Lett. 89, 027001 (2002)

9. Edwards, S.F., Anderson, P.W.: J. Phys. F 5, 965 (1975)

10. Abrikosov, A.A., Moukhin, S.I.: J. Low Temp. Phys. 33, 207 (1978)
11. Medvedev, M.V., Zaborov, A.V.: Phys. Status Solidi B 79, 379 (1977)

12. Busiello, G., De Cesare, L., Rabuffo, I.: Phys. Rev. B 28, 6463 (1983)

13. Busiello, G., De Cesare, L., Rabuffo, I.: Phys. Rev. B 29, 4189 (1984)

14. Damle, K., Sachdev, S.: Phys. Rev. B 56, 8714 (1997)

15. Damle, K., Sachdev, S.: Phys. Rev. B 57, 8307 (1998)

16. Sachdev, S.: Quantum Phase Transitions. Cambridge University Press, Cambridge (1999)

17. Tifrea, I., Bodea, D., Grosu, I., Crisan, M.: Eur. Phys. J. B 36, 377 (2003)

18. Dalidovich, D., Phillips, P.: Phys. Rev. B 63, 224503 (2001)

19. Wu, J., Phillips, P.: cond-mat/05124332 